\title{
Composition and stability of hybrid stars with hyperons and quark color-superconductivity
}

\author{
Luca Bonanno and Armen Sedrakian
}

\begin{abstract}
Institute for Theoretical Physics, J.-W. Goethe University, 60438 Frankfurt-Main, Germany e-mail: sedrakian@th.physik.uni-frankfurt.de
\end{abstract}

Received 5 August 2011 / Accepted 26 October 2011

\section{ABSTRACT}

\begin{abstract}
The recent measurement of a $1.97 \pm 0.04$ solar-mass pulsar places a stringent lower bound on the maximum mass of compact stars and therefore challenges the existence of any agents that soften the equation of state of ultra-dense matter. We investigate whether hyperons and/or quark matter can be accommodated in massive compact stars by constructing an equation of state based on a combination of phenomenological relativistic hyper-nuclear density functional and an effective model of quantum chromodynamics (the Nambu-Jona-Lasinio model). Stable configurations are obtained with $M \geq 1.97 M_{\odot}$ featuring hyper-nuclear and quark matter in color superconducting state if the equation of state of nuclear matter is stiff above the saturation density, the transition to quark matter takes place at a few times the nuclear saturation density, and the repulsive vector interactions in quark matter are substantial.
\end{abstract}

Key words. stars: neutron

\section{Introduction}

The masses of neutron stars are the most sensitive among all their parameters to the equation of state at high densities. Therefore, pulsar mass measurements provide one of the key experimental constraints on the theory of ultra-dense matter. The masses measured in the pulsar binaries are clustered around the value $1.4 M_{\odot}$ and have been consider as "canonical" for a long time. However, in recent years mounting evidence emerged in favor of substantially heavier neutron stars with $M \leq 2 M_{\odot}$. In particular, the recent discovery of a compact star with a mass of $1.97 M_{\odot}$ measured through the Shapiro delay provides an observationally "clean" lower bound on the maximum mass of a compact star (Demorest et al. 2010).

On the theoretical side it is now well-established that the emergence of new degrees of freedom at high densities softens the equation of state of matter. For example, allowing for the hyperons can reduce the maximum mass of a sequence of compact stars below the canonical mass of $1.4 M_{\odot}$. A similar reduction may occur if a deconfinement to quark matter takes place, although the softening of the equation of state in this case is less dramatic. Thus, the observation of $2 M_{\odot}$ mass neutron star is evidence that the ultra-dense matter in neutron stars cannot be soft, i.e., agents that will substantially soften the equation of state are potentially excluded.

We aim to study the equation of state of ultra-dense matter in the light of this recent constraint (Demorest et al. 2010). We investigate to which extent one can reconcile the non-nucleonic components such as hyperons and two- and three-flavor quark matter, along with their color superconductivity, with the existence of neutron stars with masses $2 M_{\odot}$. The answer(s) to the question above are of fundamental importance, because if hybrid stars featuring quark cores surrounded by a (hyper)nuclear mantle exist in nature, they could provide a unique window on the properties of quantum chromodynamics (QCD) at high baryon densities under conditions not attainable in laboratory experiments (the ultra-dense matter is in equilibrium, is charge neutral and in $\beta$-equilibrium with respect to weak interactions).
Although heavy baryons (mainly $\Sigma^{ \pm}$and $\Lambda$ hyperons) were considered even before the discovery of pulsars and their identification with the neutron stars (Ambartsumyan \& Saakyan 1960), their emergence in the cores of neutron stars is still very elusive. Treatments based on relativistic density functional methods (Glendenning 1985; Glendenning \& Moszkowski 1991; Weber 1999) predict masses that are not much larger than the canonical mass of a neutron star which clearly contradicts modern observations. Masses on the order of $\leq 1.8 M_{\odot}$ were obtained in non-relativistic phenomenological models (Balberg et al. 1999; Djapo et al. 2010), while microscopic models based on hyperon-nucleon potentials, which include the repulsive three-body forces, predict low maximal masses for hypernuclear stars (Baldo et al. 1998, 2003a; Vidaña et al. 2011).

The treatment of deconfined matter at ultra-high densities is model-dependent. We used the Nambu-Jona-Lasinio (NJL) model to describe the quark matter and its color superconductivity. The model is a non-perturbative low-energy approximation to QCD, which is anchored in the low-energy phenomenology of the hadronic spectrum. The dynamical symmetry breaking, by which quarks acquire mass, is incorporated in this model, but it lacks confinement. Our ignorance of the mechanism of confinement requires a free parameter in the theory, which can be identified with the bag constant (the latter need not be the same as in the MIT bag model). Furthermore, it can be eliminated in favor of a more physical quantity - the transition density from (hyper)nuclear to quark matter. In the low-density matter a candidate superconducting phase is the two-superconductingcolors (2SC) phase (Bailin \& Love 1984), which at sufficiently high densities transforms to the three-flavor color-flavor-locked (CFL) phase (Alford et al. 1999); our present study includes only these two phases, but we emphasize that the phase structure of matter could be more complicated (Rüster et al. 2005). The separate problem of compact stars made of strange matter with equal number of flavors of quarks (Weber \& Negreiros 2011) will not be considered here. 
Early studies of hadron-quark phase transition and quark superconductivity within the NJL model suggested that no stable stars (in the CFL phase) can be obtained within the standard parameterization of this model (see, e.g., Baldo et al. 2003b). Recently, NJL-model based stable sequences of hadron-quark stars were obtained for 2SC and CFL matter (Klähn et al. 2007; Alford et al. 2007) and a three-flavor crystalline color superconducting state (Ippolito et al. 2008). The key requirement for the existence (and stability) of these objects was the observation that the nuclear equation of state must be stiff and the quark equation of state must be supplemented by a shift (bag constant) to enable the the quark and nuclear equations of state to match (Ippolito et al. 2008). In the latter study stable crystalline color superconducting stars with masse $M \sim 2 M_{\odot}$ were obtained as "twin" configurations of purely nuclear counterparts. Subsequently, sequences of stars containing homogeneous quark matter in the 2SC and CFL phases (with maximum masses $\leq 1.8 M_{\odot}$ ) were obtained in the NJL model supplemented by a repulsive vector interaction (Pagliara \& Schaffner-Bielich 2008; Lugones et al. 2010). The importance of the vector interactions in stiffening the quark equation of state has been pointed out earlier by Klähn et al. (2007). Furthermore, the emergence of twin configurations of color-superconducting stars was confirmed in complementary studies based on variations and extensions of the NLJ model (Blaschke et al. 2010a,b; Agrawal 2010).

Massive hybrid configurations were also constructed within phenomenological parameterizations of the quark matter equation of state motivated by the MIT bag model in combination with nuclear equations of state that are moderately soft; large masses require strong quark-quark correlations in combination with color superconductivity in the CFL phase (Alford et al. 2005; Weissenborn et al. 2011).

Below we shall adopt the point of view that the nuclear equation of state needs to be stiff above the saturation density in order to obtain massive hybrid stars (Ippolito et al. 2008). With this observation as a working hypothesis we will explore the range of parameters allowing for compact stars featuring hyperonic and/or two- and three-flavor quark matter.

\section{Models}

We start with the low-density part of the equation of state, where the degrees of freedom are the nucleons and hyperons. The nuclear equation of state, as is well known, can be constructed starting from a number of principles, see, e.g., Weber (1999) and Sedrakian (2007). Below we will work with the relativistic mean-field models, which are fitted to the bulk properties of nuclear matter and hypernuclear data to describe the baryonic octet and its interactions (Glendenning \& Moszkowski 1991; Lalazissis et al. 1997). We adopt the following Walecka Lagrangian, which includes self-interacting $\sigma$-field

$$
\begin{aligned}
\mathcal{L}_{B}= & \sum_{B} \bar{\psi}_{B}\left[\gamma^{\mu}\left(\mathrm{i} \partial_{\mu}-g_{\omega B} \omega_{\mu}-\frac{1}{2} g_{\rho B} \boldsymbol{\tau} \cdot \boldsymbol{\rho}_{\mu}\right)\right. \\
& \left.-\left(m_{B}-g_{\sigma B} \sigma\right)\right] \psi_{B}+\frac{1}{2} \partial^{\mu} \sigma \partial_{\mu} \sigma-\frac{1}{2} m_{\sigma}^{2} \sigma^{2} \\
& +\frac{1}{2} m_{\omega}^{2} \omega^{\mu} \omega_{\mu}-\frac{1}{4} \rho^{\mu \nu} \cdot \boldsymbol{\rho}_{\mu \nu}+\frac{1}{2} m_{\rho}^{2} \rho^{\mu} \cdot \boldsymbol{\rho}_{\mu} \\
& -\frac{1}{3} b m_{N}\left(g_{\sigma N} \sigma\right)^{3}-\frac{1}{4} c\left(g_{\sigma N} \sigma\right)^{4} \\
& +\sum_{\mathrm{e}^{-}, \mu^{-}} \bar{\psi}_{\lambda}\left(\mathrm{i} \gamma^{\mu} \partial_{\mu}-m_{\lambda}\right) \psi_{\lambda}-\frac{1}{4} F^{\mu v} F_{\mu \nu},
\end{aligned}
$$

where the $B$-sum is over the baryonic octet $B \equiv p, n, \Lambda, \Sigma^{ \pm, 0}$, $\Xi^{-, 0}, \psi_{B}$ are the corresponding Dirac fields, whose interactions are mediated by the $\sigma$ scalar, $\omega_{\mu}$ isoscalar-vector and $\rho_{\mu}$ isovector-vector meson fields. The meson and the baryon masses correspond to their values in the vacuum, the values of nucleonmeson couplings are $g_{\sigma N} / m_{\sigma}=3.967 \mathrm{fm}, g_{\omega N} / m_{\omega}=3.244 \mathrm{fm}$, $g_{\rho N} / m_{\rho}=1.157 \mathrm{fm}$ for nucleons; the hyperon-meson couplings are obtained from these by multiplication by factors $0.6,0.658$, and 0.6 , respectively. The couplings in the self-interaction terms of the $\sigma$-field are given by $b=0.002055$ and $c=-0.002651$. The next-to-last term in Eq. (1) is the Dirac Lagrangian of leptons, $F_{\mu \nu}$ is the energy and momentum tensor of the electromagnetic field; we will not need the explicit from of other tensors in the Lagrangian (1). The parameters above correspond to the NL3 parametrization (Lalazissis et al. 1997). Computations were made also with the GM3 parameterization (Glendenning \& Moszkowski 1991), and we will comment on the differences below. The choice of this specific parametrization was made because the nucleonic matter has the stiffest equation of state compatible with the nuclear phenomenology. The mean-field pressure of the (hyper)nuclear matter can be obtained from Eq. (1) in the standard fashion (Weber 1999).

The high-density quark matter is described by an NJL Lagrangian, which is extended to include the t' Hooft interaction term $(\propto K)$ and the vector interactions $\left(\propto G_{V}\right)$

$$
\begin{aligned}
\mathcal{L}_{Q}= & \bar{\psi}\left(\mathrm{i} \gamma^{\mu} \partial_{\mu}-\hat{m}\right) \psi+G_{V}\left(\bar{\psi} \mathrm{i} \gamma^{0} \psi\right)^{2} \\
& +G_{S} \sum_{a=0}^{8}\left[\left(\bar{\psi} \lambda_{a} \psi\right)^{2}+\left(\bar{\psi} \mathrm{i} \gamma_{5} \lambda_{a} \psi\right)^{2}\right] \\
& +G_{D} \sum_{\gamma, c}\left[\bar{\psi}_{\alpha}^{a} \mathrm{i} \gamma_{5} \epsilon^{\alpha \beta \gamma} \epsilon_{a b c}\left(\psi_{C}\right)_{\beta}^{b}\right]\left[\left(\bar{\psi}_{C}\right)_{\rho}^{r} \mathrm{i} \gamma_{5} \epsilon^{\rho \sigma \gamma} \epsilon_{r s c} \psi_{\sigma}^{8}\right] \\
& -K\left\{\operatorname{det}_{f}\left[\bar{\psi}\left(1+\gamma_{5}\right) \psi\right]+\operatorname{det}_{f}\left[\bar{\psi}\left(1-\gamma_{5}\right) \psi\right]\right\},
\end{aligned}
$$

where the quark spinor fields $\psi_{\alpha}^{a}$ carry color $a=r, g, b$ and flavor ( $\alpha=u, d, s$ ) indices, the matrix of quark current masses is given by $\hat{m}=\operatorname{diag}_{f}\left(m_{u}, m_{d}, m_{s}\right), \lambda_{a}$ with $a=1, \ldots, 8$ are the GellMann matrices in the color space, and $\lambda_{0}=(2 / 3) \mathbf{1}_{\mathbf{f}}$. The charge conjugate spinors are defined as $\psi_{C}=C \bar{\psi}^{\mathrm{T}}$ and $\bar{\psi}_{C}=\psi^{\mathrm{T}} C$, where $C=\mathrm{i} \gamma^{2} \gamma^{0}$ is the charge conjugation matrix. The partition function of the system can be evaluated for the Lagrangian (2) neglecting the fluctuations beyond the mean-field (Rüster et al. 2005). To do so, one linearizes the interaction term keeping the di-quark correlations $\Delta_{c} \propto\left(\bar{\psi}_{C}\right)_{\alpha}^{a} \mathrm{i} \gamma_{5} \epsilon^{\alpha \beta c} \epsilon_{a b c} \psi_{\beta}^{b}$ and quark-antiquark correlations $\sigma_{\alpha} \propto \bar{\psi}_{\alpha}^{a} \psi_{\alpha}^{a}$. At zero temperature the pressure is given by

$$
\begin{aligned}
p= & \frac{1}{2 \pi^{2}} \sum_{i=1}^{18} \int_{0}^{\Lambda} \mathrm{d} k k^{2}\left|\epsilon_{i}\right|+4 K \sigma_{u} \sigma_{d} \sigma_{s} \\
& -\frac{1}{4 G_{D}} \sum_{c=1}^{3}\left|\Delta_{c}\right|^{2}-2 G_{s} \sum_{\alpha=1}^{3} \sigma_{\alpha}^{2}+\frac{1}{4 G_{V}}\left(2 \omega_{0}^{2}+\phi_{0}^{2}\right) \\
& +\sum_{l=\mathrm{e}^{-}, \mu^{-}} p_{l}-p_{0}-B^{*}
\end{aligned}
$$

where $\epsilon_{i}$ are the quasiparticle spectra of quarks, $\omega_{0}=$ $G_{V}\left\langle Q M\left|\psi_{u}^{\dagger} \psi_{u}+\psi_{d}^{\dagger} \psi_{d}\right| Q M\right\rangle$ and $\phi_{0}=2 G_{V}\left\langle Q M\left|\psi_{s}^{\dagger} \psi_{s}\right| Q M\right\rangle$ are the mean field expectation values of the vector mesons $\omega$ and $\phi$ in quark matter, $p_{l}$ is lepton pressure, $p_{0}$ is the vacuum pressure and $B^{*}$ is an effective bag constant. The quark chemical potentials are modified by the vector fields as follow $\hat{\mu}^{*}=\operatorname{diag}_{f}\left(\mu_{u}-\right.$ $\left.\omega_{0}, \mu_{d}-\omega_{0}, \mu_{s}-\phi_{0}\right)$. The numerical values of the parameters of the Lagrangian are $m_{u, d}=5.5 \mathrm{MeV}, m_{s}=140.7 \mathrm{MeV}$, $\Lambda=602.3 \mathrm{MeV}, G_{S} \Lambda^{2}=1.835, K \Lambda^{5}=12.36$, and $G_{D} / G_{S}=1$. 


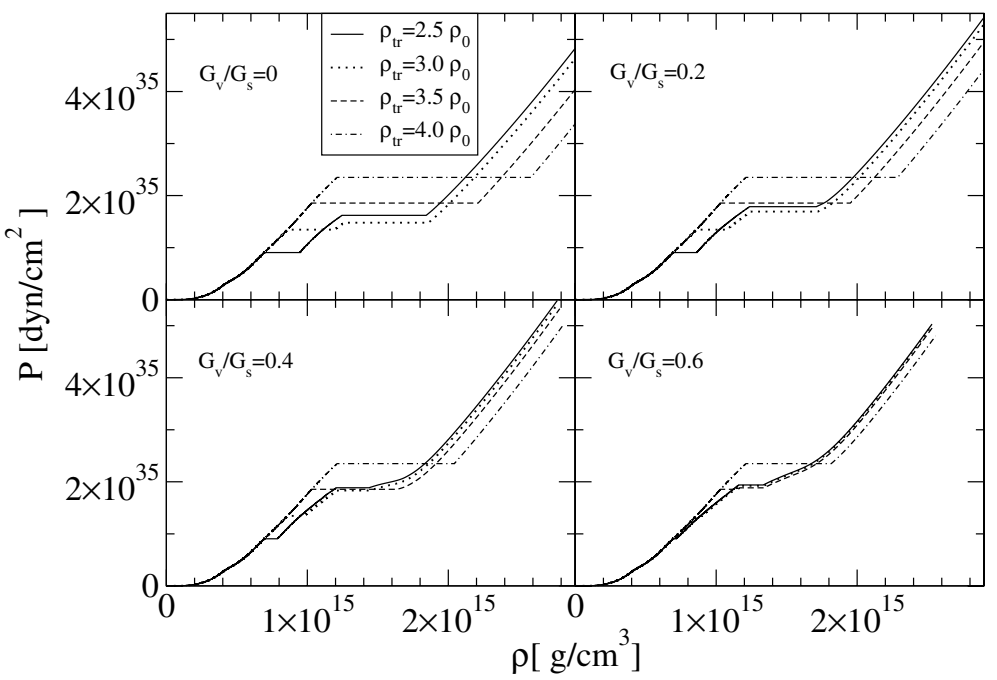

Fig. 1. Dependence of pressure on the density of matter in $\beta$-equilibrium at $T=0$ for four values of transition density from (hyper)nuclear matter to quark matter $\left(\rho_{\mathrm{tr}} / \rho_{0}=2.5,3,3.5\right.$ and 4$)$. The value of the vector coupling constant varies from zero (upper left panel) to $0.6 G_{S}$ (lower right panel).

The surface tension between the (hyper)nuclear and quark matter is not well-known, therefore we shall adopt the working hypothesis that this tension is high enough to prevent the formation of mixed phases. Then, the transition from (hyper)nuclear matter to quark matter occurs at a certain baryo-chemical potential at which the pressures of these phases are equal. This is equivalent to the condition that pressure, $P$, vs. chemical potential, $\mu$, curves for these phases cross (Maxwell construction). Thus, according to the Maxwell construction of the deconfinement phase transition, there is a jump in the density at constant pressure. However, the transition density itself cannot be fixed, because the current NJL model does not allow us to fix the low-density normalization of the pressure; (this is the consequence of the fact that this class of models does not capture the confinement feature of the QCD). For this reason we introduced above an additional "bag" parameter $B^{*}$, which allows us to vary the density at which the quark phase sets-in, thus fixing the density of deconfinement $\rho_{\text {tr }}$. The transition density increases with $B^{*}$ (as well as with the vector coupling $G_{V}$ ). For example, varying $B^{*}$ in the range $-40 \mathrm{MeV} \mathrm{fm}^{-3}$ to $50 \mathrm{MeV} \mathrm{fm}^{-3}$ we find variations in the transition density in the range $2.4 \rho_{0}<\rho_{\operatorname{tr}}<4 \rho_{0}$, where $\rho_{0}$ is the nuclear saturation density.

Before turning to the results of calculations, we briefly summarize the physical input of the underlying models. The masses and coupling in the Lagrangian (1) are constrained by the phenomenology of nuclear and hyper-nuclear matter; we will use the NL3 parameterization throughout. The parameters of the Lagrangian (2) are fixed to reproduce the observables of vacuum QCD: the pion mass $m_{\pi}=135 \mathrm{MeV}$, the kaon mass $m_{K}=497.7 \mathrm{MeV}$, the eta-prime mass $m_{\eta^{\prime}}=957.8 \mathrm{MeV}$ and the pion-decay constant $f_{\pi}=92.4 \mathrm{MeV}$. There remain two free parameters $G_{V}$ and $B^{*}$. The first one can be used to regulate the stiffness of the quark matter equation of state $\left(G_{V}=0\right.$ gives the softest equation of state), while $B^{*}$ can be varied to change the density of the phase transition to the quark phase (deconfinement). The contributions to the pressure from the condensates arises from two phases: the two-flavor 2SC phase, where $\Delta_{1}=\Delta_{2}=0$ and $\Delta_{3} \neq 0$ and the three-flavor CFL phase, where $\Delta_{1} \neq 0, \Delta_{2} \neq 0$, and $\Delta_{3} \neq 0$. The strength of the pairing field is fixed by setting $G_{D}=G_{S}$, which implies stronger pairing interaction than the one deduced from the Fierz transformation $G_{D}=0.75 G_{S}$.

\section{Results}

Figure 1 shows the equations of state of (hyper)nuclear and quark phases and their matching via Maxwell construction for several values of the parameters $G_{V}$ and $\rho_{\text {tr }}$. For low values of the $G_{V}$ parameter there are two sequential transitions from (hyper)nuclear matter to the $2 \mathrm{SC}$ phase and from $2 \mathrm{SC}$ phase to the CFL phase. As the onset density of the quark matter is shifted to higher densities, the CFL phase is expelled from the density range relevant for the phenomenology. For higher values of the $G_{V}$ parameter the density jump(s) are smaller, therefore the CFL phase can nucleate at lower densities. This tendency eventually leads to a smooth transition between the phases for $G_{V}=$ $0.6 G_{S}$, if the transition density is not too high. The t' Hooft term makes the transition from the $2 \mathrm{SC}$ to the CFL phase "smoother".

The equations of state described above were supplemented by an equation of state of the low-density crust (Baym et al. 1971). These were used as an input into the OppenheimerVolkoff equations to obtain sequences of stellar configurations. The resulting mass-radius relationship for massive stars is shown in Fig. 2 together with the largest mass measurement to date $M=$ $1.97 \pm 0.04 M_{\odot}$ (Demorest et al. 2010). Masses above the lower bound on the maximum mass are obtained for purely hadronic stars; this feature is prerequisite for finding similar stars with quark phases. Evidently only for high values of vector coupling $G_{V}$ one finds stable stars that contain (at the bifurcation from the hadronic sequence) the 2SC phase, which are followed by stars that additionally contain the CFL phase (for higher central densities). Thus we find that the stable branch of the sequence contains stars with quark matter in the 2SC and CFL phases. Similar results were obtained from the GM3 parameterization of nuclear matter if, however, the hyperons were excluded from the consideration. Therefore, a less stiff GM3 parameterization is qualitatively equivalent to the NL3 parametrization, which features the softening of the equation of state due to the hyperons.

Figure 3 summarizes several results for the masses and composition of compact stars as a function of the parameters of the 


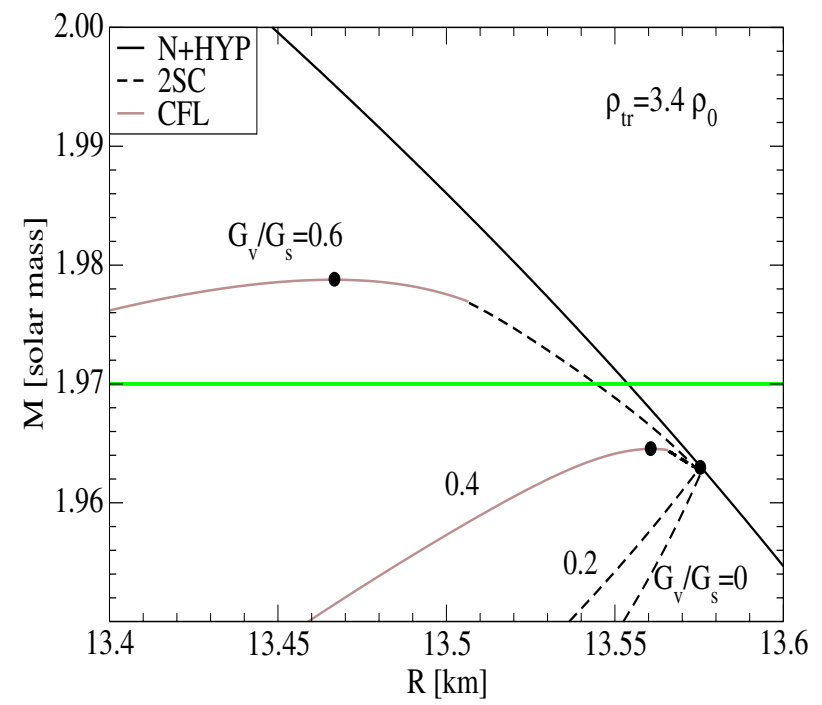

Fig. 2. Mass vs. radius for configurations with quark-hadron transition density $\rho_{\text {tr }}=3.4 \rho_{0}$ for four values of vector coupling $G_{V} / G_{S}=$ $0,0.2,0.4,0.6$. The purely hadronic sequence (i.e. the sequence that includes nucleons and hyperons) is shown by black solid line. The dashed lines and the gray solid lines show the branches where the 2SC and CFL quark phases are present. The filled circles mark the maximum masses of the sequences. The horizontal line shows the largest mass measurement to date (Demorest et al. 2010).

model $G_{V}$ and $\rho_{\mathrm{tr}}$. First, it shows the tracks of constant maximum mass compact stars within the parameter space. The decrease of these lines with increasing vector coupling reflects the fact that non-zero vector coupling stiffens the equation state. In other words, to obtain a given maximum mass one can admit a small amount of soft quark matter with vanishing vector coupling by choosing a high transition density; the same result is obtained with a low transition density, but strong vector coupling, i.e., a stiffer quark equation of state. For low transition densities one finds 2SC matter in stars, which means that weaker vector couplings slightly disfavor 2SC matter. Substantial CFL cores appear in configurations for strong vector coupling and almost independent of the transition density (nearly vertical dashed lines with $\delta \sim 0.1$ in Fig. 3). Note that for a high transition density there is a direct transition from hyper-nuclear to the CFL phase. For transition densities blow $3.5 \rho_{0}$ a $2 \mathrm{SC}$ layer emerges that separates these phases. On the other hand, weak vector couplings and low transition densities produce stars with a 2SC phase only.

In Fig. 4 we show the baryon density profiles of the maximum-mass stars with a transition density of $\rho_{\text {tr }}=2.5 \rho_{0}$. For soft quark matter $G_{V}=0$ we obtain quark matter only in the 2SC phase, which extends up to about $1 / 3$ of the star radius. Increasing $G_{V}$ has the effect of shifting the $2 \mathrm{SC}$ phase to larger radii, whereas the CFL phase develops at the center. For the strongest coupling studied, all phases are present in the maximum mass star, with the combined 2SC and CFL paired quark matter confined within a radius that is about the half of the star. Note that a similar internal structure is obtained for crystalline color-superconducting stars, where quark matter is confined within a radius of $\sim 7 \mathrm{~km}$ for maximum mass stars with a radius $\sim 12 \mathrm{~km}$ (Knippel \& Sedrakian 2009).

\section{Conclusion}

Massive neutron stars are likely to develop cores composed of deconfined quark matter, which should be in one of the color

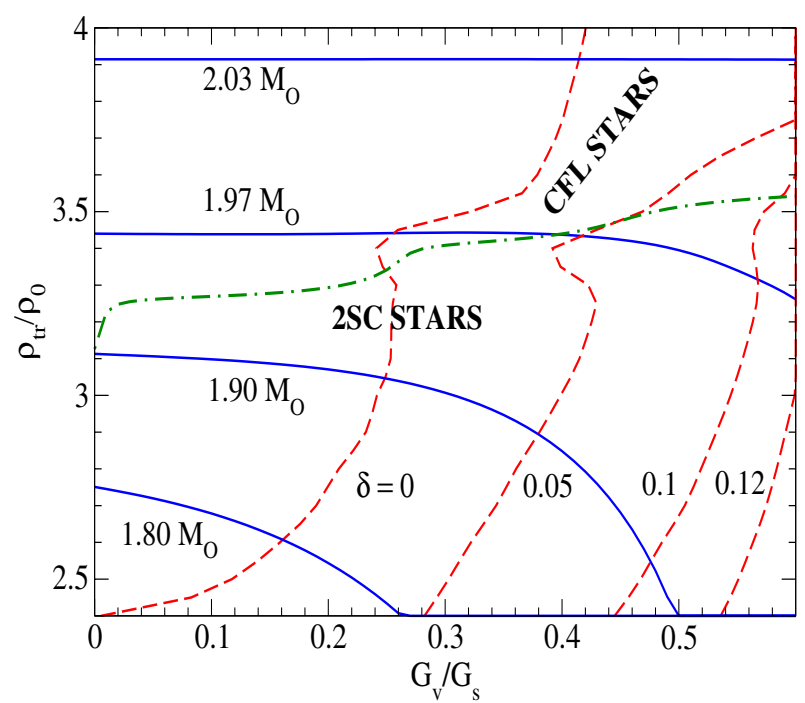

Fig. 3. Properties of the stars as a function of the free parameters $G_{V}$ and $\rho_{\mathrm{tr}}$. The solid lines (blue online) show the maximum mass configurations realized for the pair of parameters $G_{V}$ and $\rho_{\mathrm{tr}}$. The dashed (red online) curves show the amount of CFL matter in the configurations via the ratio $\delta=R_{\mathrm{CFL}} / R$, where $R_{\mathrm{CFL}}$ is the radius of the CFL core, $R$ is the star radius. The parameter space to the right from $\delta=0$ line produces CFL stars. The parameter space below the dashed-dotted (green online) curve corresponds to stars containing 2SC matter.

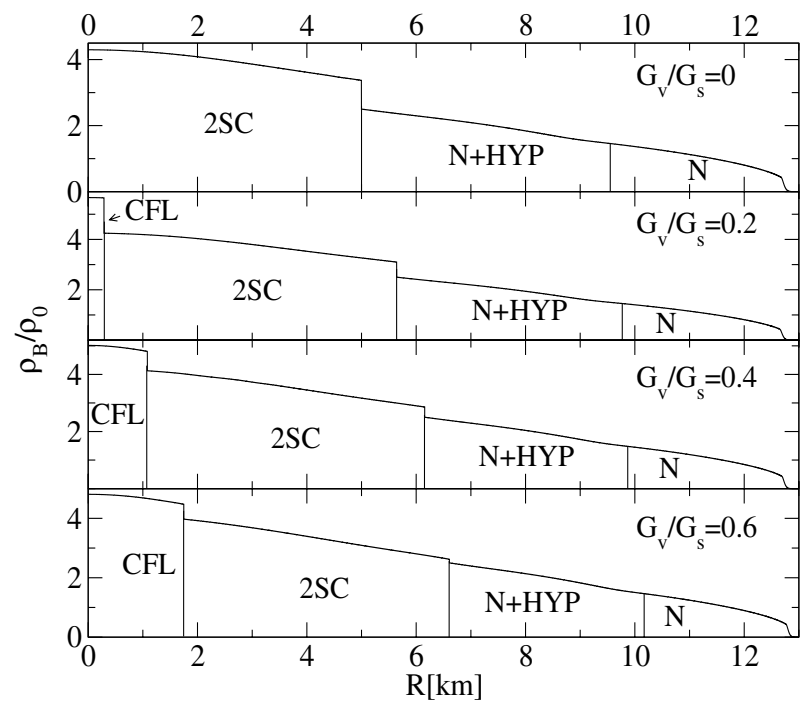

Fig. 4. Density profiles of the maximum mass configurations with the quark-hadron transition density $\rho_{\mathrm{tr}}=2.5 \rho_{0}$. The vector coupling constant varies from zero (upper panel) to $0.6 G_{S}$ (lower panel). The hypernuclear and nuclear phases are marked by $\mathrm{N}+\mathrm{HYP}$ and $\mathrm{N}$ respectively.

superconducting phases. We have constructed an equation of state of such matter on the basis of relativistic mean-field nuclear functional at low densities and effective NJL model of quark matter supplemented by the t' Hooft and vector interactions. Non-rotating spherically symmetric configuration with maximum masses $\sim 2 M_{\odot}$ were studied by solving the OppenheimerVolkoff equations and were compared to the recent lower bound on the maximum mass of a neutron star. Our equation of state contains two free parameters: the transition density from (hyper)nuclear matter to quark matter and the vector coupling of quarks. We have demonstrated that stable configurations featuring color superconducting matter can be constructed if vector interactions are included in the quark equation of state. A 
prerequisite for this is a stiff nuclear equation of state. We find that the NL3 parameterization with hyperons or GM3 parameterization with nucleons only are sufficiently stiff to produce maximum masses above the lower bound. Inclusion of quark degrees of freedom softens these equations of state; the required lower bound can still be achieved because the vector interactions in the quark matter can stiffen the quark equation of state.

To conclude, relativistic hypernuclear Lagrangians, which predict stiff hypernuclear equations of state above saturation density, enable us to construct stable configurations with masses equal and above the measured 1.97 solar-mass star. Our configuration have "exotic" matter in their interiors in the form of hyperons and quark matter, of which the quark matter is color-superconducting in the two-flavor $2 \mathrm{SC}$ and/or three-flavor CFL phases. The choice of parameters in our models geared toward producing stiff equations of states above the saturation density. Observations of pulsars with larger masses (above $2 M_{\odot}$ ) will seriously challenge our current understanding of dense matter physics above nuclear saturation.

Acknowledgements. This work was supported in part by the Helmholtz International Center for FAIR within the framework of the LOEWE (Landesoffensive zur Entwicklung Wissenschaftlich-Ökonomischer Exzellenz) program launched by the State of Hesse and by the Research Networking Programme of the European Science Foundation "Compstar".

\section{References}

Agrawal, B. K. 2010, Phys. Rev. D, 81, 023009

Alford, M., Rajagopal, K., \& Wilczek, F. 1999, Nucl. Phys. B, 537, 443
Alford, M., Braby, M., Paris, M., \& Reddy, S. 2005, ApJ, 629, 969

Alford, M., Blaschke, D., Drago, A., et al. 2007, Nature, 445

Ambartsumyan, V. A., \& Saakyan, G. S. 1960, AZh., 37, 193

Bailin, D., \& Love, A. 1984, Phys. Rep., 10 7, 325

Balberg, S., Lichtenstadt, I., \& Cook, G. B. 1999, ApJS, 121, 515

Baldo M., Burgio F., \& Schulze H.-J. 1998, Phys. Rev. C, 58, 3688

Baldo, M., Burgio, G. F., \& Schulze, H. 2003a [arXiv:astro-ph/0312446]

Baldo, M., Buballa, M., Burgio, G. F., et al. 2003b, Phys. Lett. B, 562, 153

Baym, G., Pethick, C., \& Sutherland, P. 1971, ApJ, 170, 299

Blaschke, D., Klähn, T., Łastowiecki, R., \& Sandin, F. 2010a, J. Phys. G Nucl. Phys., 37, 094063

Blaschke, D., Berdermann, J., \& Łastowiecki, R. 2010b, Prog. Theor. Phys. Suppl., 186, 81

Demorest, P. B., Pennucci, T., Ransom, S. M., Roberts, M. S. E., \& Hessels, J. W. T. 2010, Nature, 467, 1081

Djapo, H., Schaefer, B.-J., \& Wambach, J. 2010, Phys. Rev. C, 81, 035803

Glendenning, N. K. 1985, ApJ, 293, 470

Glendenning, N. K., \& Moszkowski, S. A. 1991, Phys. Rev. Lett., 67, 2414

Ippolito, N. D., Ruggieri, M., Rischke, D. H., Sedrakian, A., \& Weber, F. 2008, Phys. Rev. D, 77, 023004

Klähn, T., Blaschke, D., Sandin, F., et al. 2007, Phys. Lett. B, 654, 170

Knippel, B., \& Sedrakian, A. 2009, Phys. Rev. D, 79, 083007

Lalazissis, G. A., König, J., \& Ring, P. 1997, Phys. Rev. C, 55, 540

Lugones, G., Do Carmo, T. A. S., Grunfeld, A. G., \& Scoccola, N. N. 2010, Phys. Rev. D, 81, 085012

Pagliara, G., \& Schaffner-Bielich, J. 2008, Phys. Rev. D, 77, 063004

Rüster, S. B., Werth, V., Buballa, M., Shovkovy, I. A., \& Rischke, D. H. 2005, Phys. Rev. D, 72, 034004

Sedrakian, A. 2007, Prog. Part. Nuc. Phys., 58, 168

Weber, F. 1999, Pulsars as astrophysical laboratories for nuclear and particle physics (Bristol, UK: Institute of Physics)

Weber, F., \& Negreiros, R. 2011, AIP Conf. Ser., 1354, 13

Weissenborn, S., Sagert, I., Pagliara, G., Hempel, M., \& Schaffner-Bielich, J. 2011, ApJ, 740, L14

Vidaña, I., Logoteta, D., Providência, C., Polls, A., \& Bombaci, I. 2011, Europhys. Lett., 94, 11002 